\title{
Benjamini-Schramm convergence and the distribution of chromatic roots for sparse graphs
}

\author{
Miklós Abért and Tamás Hubai
}

May 20, 2013

\begin{abstract}
We define the chromatic measure of a finite simple graph as the uniform distribution on its chromatic roots. We show that for a BenjaminiSchramm convergent sequence of finite graphs, the chromatic measures converge in holomorphic moments.

As a corollary, for a convergent sequence of finite graphs, we prove that the normalized log of the chromatic polynomial converges to an analytic function outside a bounded disc. This generalizes a recent result of Borgs, Chayes, Kahn and Lovász, who proved convergence at large enough positive integers and answers a question of Borgs.

Our methods also lead to explicit estimates on the number of proper colorings of graphs with large girth.
\end{abstract}

\section{Introduction}

Let $G$ be a finite undirected graph without multiple edges and loops. A map $f: V(G) \rightarrow\{1, \ldots, q\}$ is a proper coloring if for all edges $(x, y) \in E(G)$ we have $f(x) \neq f(y)$. For a positive integer $q$ let $\operatorname{ch}_{G}(q)$ denote the number of proper colorings of $G$ with $q$ colors. Then $\operatorname{ch}_{G}$ is a polynomial in $q$, called the chromatic polynomial of $G$. The complex roots of $\operatorname{ch}_{G}$ are called the chromatic roots of $G$.

The study of chromatic polynomials and its roots has been initiated by Birkhoff. Since then, the subject has gotten considerable interest, partially because of its connection to statistical mechanics. In particular, the chromatic roots control the behaviour of the antiferromagnetic Potts model at zero temperature. For a survey on the subject see [13].

For a finite graph $G$, a finite rooted graph $\alpha$ and a positive integer $R$, let $\mathbf{P}(G, \alpha, R)$ denote the probability that the $R$-ball centered at a uniform random vertex of $G$ is isomorphic to $\alpha$. We say that a graph sequence $\left(G_{n}\right)$ of bounded degree is Benjamini-Schramm convergent if for all finite rooted graphs $\alpha$ and $R>0$, the probabilities $\mathbf{P}\left(G_{n}, \alpha, R\right)$ converge (see [3]). This means that one can not statistically distinguish $G_{n}$ and $G_{n^{\prime}}$ for large $n$ and $n^{\prime}$ by sampling them from a random vertex with a fixed radius of sight. An example (that is regularly used in statistical physics) is to approximate the infinite lattice $\mathbb{Z}^{d}$ by 
bricks with all the side lengths tending to infinity. More generally, amenable vertex transitive graphs can be obtained as the Benjamini-Schramm limits of their Følner sequences.

For a simple graph $G$ let $\mu_{G}$, the chromatic measure of $G$ denote the uniform distribution on its chromatic roots. By a theorem of Sokal [12, $\mu_{G}$ is supported on the open disc of radius $C d$ around 0 , denoted by

$$
D=B(0, C d)
$$

where $d$ is the maximal degree of $G$ and $C<8$ is an absolute constant.

Theorem 1.1 Let $\left(G_{n}\right)$ be a Benjamini-Schramm convergent graph sequence of absolute degree bound $d$, and $\widetilde{D}$ an open neighborhood of the closed disc $\bar{D}$. Then for every holomorphic function $f: \widetilde{D} \rightarrow \mathbb{C}$, the sequence

$$
\int_{D} f(z) d \mu_{G_{n}}(z)
$$

converges.

Let ln denote the principal branch of the complex logarithm function. For a simple graph $G$ and $z \in \mathbb{C}$ let

$$
\mathrm{t}_{G}(z)=\frac{\ln \operatorname{ch}_{G}(z)}{|V(G)|}
$$

where this is well-defined. In statistical mechanics, $\mathrm{t}_{G}(z)$ is called the entropy per vertex or the free energy at $z$. In their recent paper [4, Borgs, Chayes, Kahn and Lovász proved that if $\left(G_{n}\right)$ is a Benjamini-Schramm convergent graph sequence of absolute degree bound $d$, then $\mathrm{t}_{G_{n}}(q)$ converges for every positive integer $q>2 d$. Theorem 1.1 yields the following.

Theorem 1.2 Let $\left(G_{n}\right)$ be a Benjamini-Schramm convergent graph sequence of absolute degree bound d with $\left|V\left(G_{n}\right)\right| \rightarrow \infty$. Then $\mathrm{t}_{G_{n}}(z)$ converges to a real analytic function on $\mathbb{C} \backslash \bar{D}$.

In particular, $\mathrm{t}_{G_{n}}(z)$ converges for all $z \in \mathbb{C} \backslash \bar{D}$. Theorem 1.2 answers a question of Borgs [2, Problem 2] who asked under which circumstances the entropy per vertex has a limit and whether this limit is analytic in $1 / z$. Note that for an amenable quasi-transitive graph and its Følner sequences, this was shown to hold in $[9$.

To prove Theorem 1.1 we show that for a finite graph $G$ and for every $k$, the number

$$
p_{k}(G)=|V(G)| \int_{D} z^{k} d \mu_{G}(z)
$$


can be expressed as a fixed linear combination of $\operatorname{hom}(H, G)$ where the $H$ are connected finite graphs and $\operatorname{hom}(H, G)$ denotes the number of graph homomorphisms from $H$ to $G$. Since a sequence of graphs $G_{n}$ of bounded degree is Benjamini-Schramm convergent if and only if

$$
\frac{\operatorname{hom}\left(H, G_{n}\right)}{\left|V\left(G_{n}\right)\right|}
$$

converges for all connected graphs $H$. This gives convergence of all the holomorphic moments of $\mu_{G_{n}}$, and this is equivalent to Theorem 1.1. For instance, for the fourth moment we get

$$
\begin{aligned}
& p_{4}(G)=-\frac{1}{3} \operatorname{hom}(\bullet \cdot G)+\frac{4}{3} \operatorname{hom}(\bullet \bullet \cdot, G)-\frac{1}{2} \operatorname{hom}(\nabla, G)+\frac{1}{3} \operatorname{hom}(\bullet, G)+ \\
& \operatorname{hom}(\bullet \cdot G)-\frac{1}{2} \operatorname{hom}(\cdot \bullet, G)+\operatorname{hom}(\not \triangleright, G)-\frac{1}{3} \operatorname{hom}(\&, G)-
\end{aligned}
$$

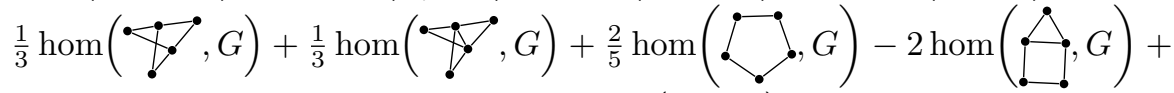

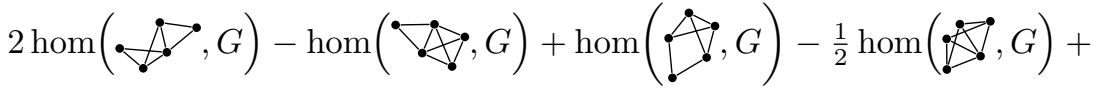

$$
\begin{aligned}
& \frac{1}{3} \operatorname{hom}(\leftrightarrow, G)-\frac{1}{30} \operatorname{hom}(\notin 0),
\end{aligned}
$$

One could speculate that assuming Benjamini-Schramm convergence of $G_{n}$, maybe the complex measures $\mu_{G_{n}}$ themselves will weakly converge. That is, Theorem 1.1 would hold for any continuous real function on $D$ or, equivalently, convergence would hold in all the moments

$$
\int_{D} z^{k} \bar{z}^{j} d \mu_{G_{n}}(z)
$$

However, this is not true in general, as the following easy counterexample shows. Let $P_{n}$ denote the path of length $n$ and let $C_{n}$ denote the cycle of length $n$. Then $P_{n}$ and $C_{n}$ converge to the same object, the infinite rooted path, while we have

$$
\operatorname{ch}_{P_{n}}(z)=z(z-1)^{n-1} \text { and } \operatorname{ch}_{C_{n}}(z)=(z-1)^{n}+(-1)^{n}(z-1) .
$$

Thus, the weak limit of $\mu_{P_{n}}$ is the Dirac measure on 1 and the weak limit of $\mu_{C_{n}}$ is the normalized Lebesgue measure on the unit circle centered at 1.

Still, using Theorem 1.1, we are able to prove the weak convergence of $\mu_{G_{n}}$ for some natural sequences of graphs. For example, let $T_{n}=C_{4} \times P_{n}$ denote the $4 \times n$ tube, i.e. the cartesian product of the 4 -cycle with a path on $n$ vertices. $T_{n}$ is a 4-regular graph except at the ends of the tube.

Proposition 1.3 The chromatic measures $\mu_{T_{n}}$ weakly converge.

The proof is as follows: as Salas and Sokal [1] showed, the pointwise limit $X$ of supports of $\mu_{T_{n}}$ is part of a particular algebraic curve, so any subsequential weak limit is supported on $X$. The complement of $X$ is connected, so by 
Mergelyan's theorem $[8$, every continuous function on $X$ can be uniformly approximated by polynomials. Using Theorem 1.1 this yields weak convergence of $\mu_{T_{n}}$. See Section 4 for details.

In this case one can use the so-called transfer matrix method to control the support of the chromatic measures (see [11] for various models related to the square lattice). In general, even for models of the square lattice, the complement of the limiting set may not be connected, and hence one can not invoke Mergelyan's theorem. It is expected, however, that for any model where the transfer matrix method can be used, the chromatic measures do converge weakly.

Another naturally interesting case is when the girth of $G$ (the minimal size of a cycle) is large. One can show that

$$
\int_{D} z^{k} d \mu(z)=\frac{|E(G)|}{|V(G)|} \quad(1 \leq k \leq \operatorname{girth}(G)-2)
$$

that is, the moments are all the same until the girth is reached (see Lemma 5.2 . This implies that for a sequence of $d$-regular graphs $G_{n}$ with girth tending to infinity, the limit of the free entropy

$$
\lim _{n \rightarrow \infty} \mathrm{t}_{G_{n}}(z)=\ln q+\frac{d}{2} \ln \left(1-\frac{1}{q}\right)
$$

for $q>C d$. This is one of the main results in [1. Note that their proof works for $q>d+1$, while our approach only works for $q>C d$. The advantage of our approach is that it gives an explicit estimate on the number of proper colorings of large girth graphs.

Theorem 1.4 Let $G$ be a finite graph of girth $g$ and maximal degree $d$. Then for all $q>C d$ we have

$$
\left|\frac{\ln \operatorname{ch}_{G}(q)}{|V(G)|}-\left(\ln q+\frac{|E(G)|}{|V(G)|} \ln \left(1-\frac{1}{q}\right)\right)\right| \leq 2 \frac{(C d / q)^{g-1}}{1-C d / q} .
$$

When $G$ is $d$-regular with asymptotically maximal girth, i.e. $g=c \ln |V(G)|$, this yields

$$
\left|\frac{\ln \operatorname{ch}_{G}(q)}{|V(G)|}-\left(\ln q+\frac{d}{2} \ln \left(1-\frac{1}{q}\right)\right)\right| \leq O\left(|V(G)|^{-c^{\prime}}\right)
$$

for some explicit constant $c^{\prime}>0$. Counting the number of proper colorings of random $d$-regular graphs have been considered in [1. These graphs do not have logarithmic girth, but they have few shorter cycles, so one can obtain a similar result for them.

Here we wish to raise attention to an interesting phenomenon, of which we only have some computational evidence. We have computed the chromatic measures of several 3-regular large girth graphs and surprisingly, it looks like one may also get weak convergence of chromatic measures. 


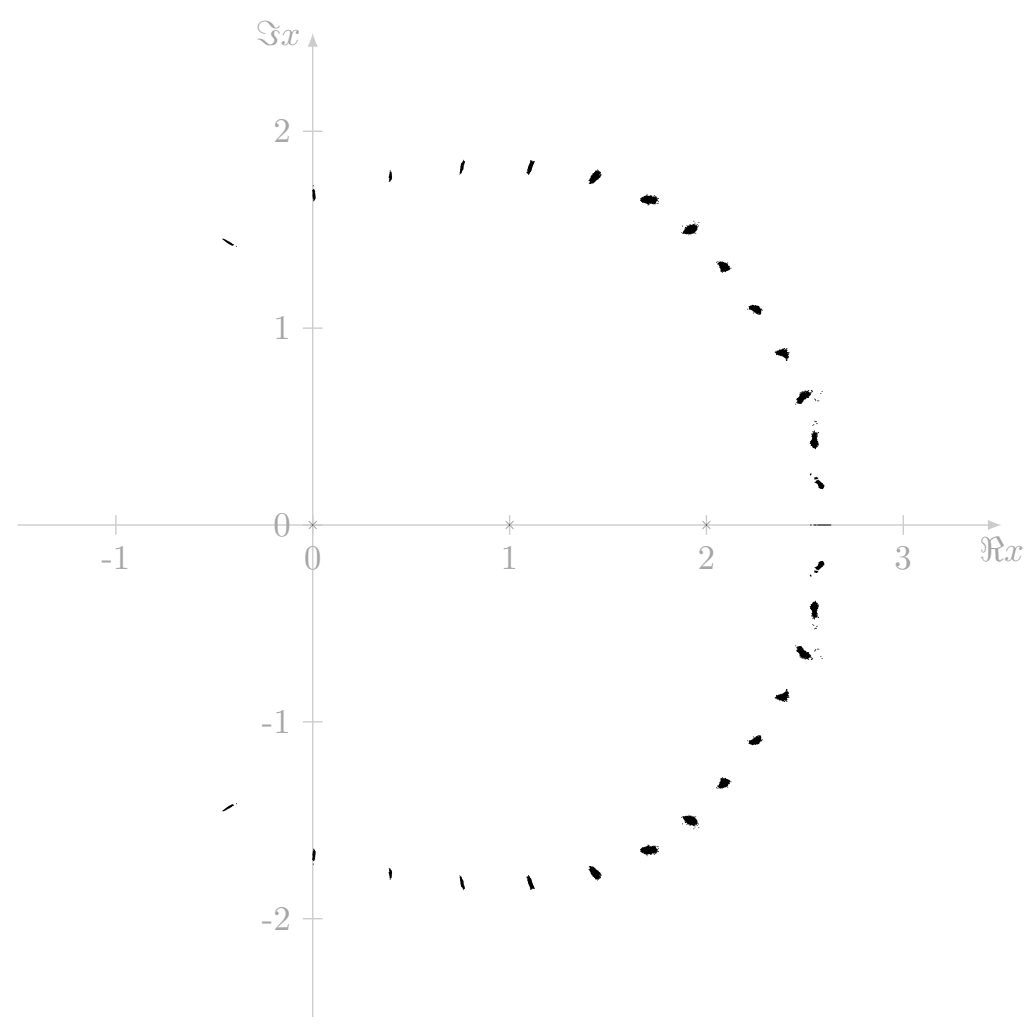

Figure 1: Chromatic roots of the 30368 cubic graphs of size 32 and girth 7

Problem 1.5 Let $G_{n}$ be a sequence of d-regular graphs with girth tending to infinity. Does $\mu_{G_{n}}$ weakly converge?

This would be interesting because one could consider the limit as the 'chromatic measure of the $d$-regular infinite tree'. Observe that any subsequential weak limit $\mu$ of $\mu_{G_{n}}$ satisfies

$$
\int_{D} z^{k} d \mu(z)=\frac{d}{2} \quad(k \geq 1)
$$

that is, the holomorphic moments of $\mu$ are independent of $k$. While Figure 1 looks very promising, one misleading aspect of it may be that 3-regular graphs having 32 vertices and (maximal possible) girth 7 may exhibit structural restrictions that are much stronger than just large girth.

It would be interesting to generalize the results of this paper to the Tutte polynomial $T_{G}(x, y)$. This two-variable polynomial encodes a lot of interesting 
invariants of $G$. For instance, $T_{G}(z, 0)=\operatorname{ch}_{G}(z), T_{G}(2,1)$ counts the number of forests, $T_{G}(1,1)$ is the number of spanning trees and $T_{G}(1,2)$ is the number of connected spanning subgraphs. By a result of Lyons [7, we know that

$$
\frac{\log T_{G_{n}}(1,1)}{\left|V\left(G_{n}\right)\right|}
$$

converges for a Benjamini-Schramm convergent sequence of graphs $G_{n}$ of bounded degree. Also, in [4] it is shown that the same holds at the places $(q, y)$ where $0 \leq y<1$ and $q$ is large enough in terms of the maximal degree. It would be interesting to see whether this also holds at other places. The places $(2,1)$ and $(1,2)$ would be good test points as they have a natural combinatorial meaning. Also, it is not clear whether Theorem 1.1 holds for $p_{G}(z)=T_{G}\left(z, y_{0}\right)$ for all fixed $y_{0}$. Note that even for the chromatic polynomial, in general, the above log convergence will not hold, for instance at $(2,0)$, because cycles of even and odd length converge to the same limit, but even cycles have a proper 2-coloring, while odd cycles do not. This may not be so surprising, since $T_{G}(2,0) \leq 2^{c(G)}$ where $c(G)$ is the number of components of $G$. So for a nontrivial graph sequence $G_{n}$, $T_{G_{n}}(2,0)$ is subexponential in $\left|V\left(G_{n}\right)\right|$, which points to the proximity of roots of $T_{G_{n}}$. To apply Theorem 1.1 in its present form, one needs that some small neighbourhood of the place is sparse in terms of roots.

Remark. Note that recently Csikvári and Frenkel [5] generalized Theorem 1.2 to a large class of graph polynomials, including the Tutte polynomial. In particular, they show that convergence holds for the normalized log of $T(x, y)$ where $x, y$ have large enough absolute value.

Acknowledgements. The authors thank László Lovász who introduced them to 4, Gábor Halász who raised their attention to the relevance of Mergelyan's theorem and Lewis Bowen who pointed out some inconsistencies of notation in a previous version of this paper. This work was partially supported by the MTA Rényi "Lendület" Groups and Graphs Research Group.

\section{Preliminaries}

For a simple graph $H$ on $n$ vertices let the number of legal colorings of $H$ with $q$ colors be denoted by $\operatorname{ch}_{H}(q)$. Then for any edge $e$ of $H$ the following recursion holds:

$$
\operatorname{ch}_{H}(q)=\operatorname{ch}_{H \backslash e}(q)-\operatorname{ch}_{H / e}(q)
$$

where $H \backslash e$ is obtained from $H$ by deleting $e$ and $H / e$ is obtained by gluing the endpoints of $e$ and erasing multiple edges and loops. This implies that $\mathrm{ch}_{H}$ is a polynomial of degree $n$ in $q$ with integer coefficients, called the chromatic polynomial of $G$ and that the above recursion holds for the polynomials themselves. It also follows that the constant coefficient of $\mathrm{ch}_{H}$ is zero and its main 
coefficient is 1 . So, we can write

$$
\begin{aligned}
\operatorname{ch}_{H}(z) & =z^{n}-e_{1}(H) z^{n-1}+\ldots+(-1)^{k} e_{k}(H) z^{n-k}+\ldots+(-1)^{n-1} e_{n-1}(H) z= \\
& =\prod_{i=1}^{n}\left(z-\lambda_{i}(H)\right) .
\end{aligned}
$$

The $e_{k}(H)$ are called the chromatic coefficients of $H$ and $\lambda_{i}(H)$ are its chromatic roots. For $k \geq 0$ let

$$
p_{k}(H)=\sum_{i=1}^{n} \lambda_{i}^{k}(H) .
$$

The Newton identities establish connections between the roots and coefficients of a polynomial. In this paper we will use the following version:

$$
p_{k}=(-1)^{k-1} k e_{k}+\sum_{i=1}^{k-1}(-1)^{k-i-1} p_{i} e_{k-i} .
$$

Let $H, G$ be simple graphs. A map $f: V(H) \rightarrow V(G)$ is a homomorphism if for all edges $(x, y) \in E(H)$ we have $(f(x), f(y)) \in E(G)$. We denote the number of homomorphisms from $H$ to $G$ by hom $(H, G)$. The quantity hom $(H, G)$ is nice to work with, mainly because of the following property.

Lemma 2.1 Let $H$ be the disjoint union of $H_{1}$ and $H_{2}$. Then

$$
\operatorname{hom}(H, G)=\operatorname{hom}\left(H_{1}, G\right) \operatorname{hom}\left(H_{2}, G\right)
$$

for all simple graphs $G$.

We leave the proof to the reader.

For a random rooted graph $G$, a finite rooted graph $\alpha$ and a positive integer $R$, let $\mathbf{P}(G, \alpha, R)$ denote the probability that the $R$-ball centered at the root of $G$ is isomorphic to $\alpha$. Analogously, for an unrooted finite graph $G$, let $\mathbf{P}(G, \alpha, R)$ denote the probability that the $R$-ball centered at a uniform random vertex of $G$ is isomorphic to $\alpha$. A graph sequence $G_{n}$ has bounded degree if there is an absolute upper bound on the degrees of vertices of $G_{n}$.

A graph sequence $\left(G_{n}\right)$ of bounded degree is Benjamini-Schramm convergent if for all finite rooted graphs $\alpha$ and $R>0$ the probabilities $\mathbf{P}\left(G_{n}, \alpha, R\right)$ converge.

The limit of a Benjamini-Schramm convergent sequence of graphs is the random rooted graph $G$ satisfying

$$
\mathbf{P}(G, \alpha, R)=\lim _{n \rightarrow \infty} \mathbf{P}\left(G_{n}, \alpha, R\right)
$$

for all $R>0$ and $\alpha$. It is easy to see that $G$ is well defined. In the most transparent case, $G$ is just one graph, which then has to be vertex transitive. For instance, the $d$ dimensional lattice

$$
\mathbb{Z}^{d}=\lim _{n \rightarrow \infty}(\mathbb{Z} / n \mathbb{Z})^{d}=\lim _{n \rightarrow \infty} B_{n}^{d}
$$


where $(\mathbb{Z} / n \mathbb{Z})^{d}$ is the $d$ dimensional torus and $B_{n}^{d}$ is the box of side length $n$ in $\mathbb{Z}^{d}$. The same way, one can obtain any connected vertex transitive amenable graph as a limit. Let $G$ be a connected vertex transitive graph of bounded degree. A sequence of connected subgraphs $F_{n}$ of $G$ is a Følner sequence, if

$$
\lim _{n \rightarrow \infty} \frac{\left|\partial F_{n}\right|}{\left|V\left(F_{n}\right)\right|}=0
$$

where $\partial F_{n}$ denotes the external vertex boundary of $F_{n}$. Note that $G$ is amenable if and only if it has a Følner sequence. It is easy to show that any connected vertex transitive amenable graph is the Benjamini-Schramm limit of its Følner sequences.

Let us consider now the $d$-regular tree $T_{d}$, which is in many senses the farthest possible from being amenable. One can obtain $T_{d}$ as the limit of finite graphs, but it is worth to point out that $T_{d}$ can not be obtained as a limit of finite trees. Indeed, the expected degrees of finite trees are approximately 2 and this passes on to their limits. It is a good exercise to understand what the limit of the balls in $T_{d}$ is (it is a fixed tree where the root is random). The right way to approximate $T_{d}$ in Benjamini-Schramm convergence is to take finite $d$-regular graphs $G_{n}$ with girth tending to infinity.

Benjamini-Schramm convergence can also be expressed in terms of graph homomorphisms using the following lemma (see [6], Proposition 5.6).

Lemma 2.2 Let $G_{n}$ be a graph sequence of bounded degree. Then $G_{n}$ is BenjaminiSchramm convergent if and only if for every finite connected graph $H$, the limit

$$
\lim _{n \rightarrow \infty} \frac{\operatorname{hom}\left(H, G_{n}\right)}{\left|V\left(G_{n}\right)\right|}
$$

exists.

Note that one needs connectedness in Lemma 2.2 as $\operatorname{hom}(H, G)$ may be the order of $|V(G)|^{c}$ where $c$ is the number of components in $H$.

\section{Expressing moments from homomorphisms}

In this section we give an explicit formula for the holomorpic moments of the chromatic measure in terms of graphs homomorphisms.

For a finite, simple graph $G$ let $\mathcal{P}(G)$ be defined as the set of partitions of $V(G)$ where no edge of $G$ connects two vertices in the same class. A partition $P \in \mathcal{P}(G)$ can be considered as a surjective homomorphism from $G$ to the simple graph $G / P$ obtained by contracting each class of $P$ and erasing multiple edges. For simple graphs $G$ and $T$ let

$$
\mathcal{P}(G, T)=\{P \in \mathcal{P}(G) \mid G / P \cong T\}
$$


be the collection of partitions of $G$ with quotient isomorphic to $T$. For $P \in \mathcal{P}(G)$ let

$$
\|P\|=\prod_{p \in P}(|p|-1) !
$$

where $p \in P$ runs through the $P$-classes.

Let $\operatorname{Aut}(G)$ denote the automorphism group of $G$. Let $\mathcal{G}(k)$ denote the set of graphs without isolated vertices, where the number of vertices minus the number of components equals $k$ and let

$$
\mathcal{G}(\leq k)=\cup_{j \leq k} \mathcal{G}(j) .
$$

Note that $\mathcal{G}(\leq k)$ is a finite set.

Base parameters. Now we introduce a sequence of parameters that will connect moments with chromatic coefficients. For a simple graph $T$ and $k>0$ let

$$
c_{k}(T)=\sum_{G \in \mathcal{G}(k)} \frac{(-1)^{|E(G)|+|V(G)|+|V(T)|+k}}{|\operatorname{Aut}(G)|} \sum_{P \in \mathcal{P}(G, T)}\|P\| .
$$

It turns out that these parameters allow us to express $e_{k}(H)$ in a nice way.

Lemma 3.1 Let $H$ be a simple graph. Then we have

$$
e_{k}(H)=\sum_{T \in \mathcal{G}(\leq k)} c_{k}(T) \operatorname{hom}(T, H) .
$$

Proof. We derive the lemma from two easy claims. Let $\operatorname{inj}(G, H)$ denote the number of injective homomorphisms from $G$ to $H$.

Claim 1. We have

$$
e_{k}(H)=\sum_{G \in \mathcal{G}(k)} \frac{(-1)^{|E(G)|+k}}{|\operatorname{Aut}(G)|} \operatorname{inj}(G, H) .
$$

To see this, we use the following identity, that is sometimes used as a definition.

$$
\operatorname{ch}_{H}(z)=\sum_{\substack{G \subseteq H \\ \text { spanning }}}(-1)^{|E(G)|} z^{c(G)}
$$

where $c(G)$ is the number of connected components in the spanning subgraph $G$. It is enough to prove this for positive integer values of $z$. In this case, there are exactly $z^{c(G)}$ colorings that violate the legal coloring constraint for all edges of $G$, and the equation follows from the inclusion-exclusion principle.

The value of $e_{k}(H)$ is $(-1)^{k}$ times the coefficient of $z^{n-k}$, which contains the terms where $c(G)=n-k$, or equivalently, where the graph $G$, when erasing its isolated vertices, is in $\mathcal{G}_{k}$. A graph $G$ is counted as many times as it appears in $H$ as a spanning subgraph, which equals $\operatorname{inj}(G, H) /|\operatorname{Aut}(G)|$. Claim 1 is proved. 
Claim 2. Let $G \in \mathcal{G}(k)$ and let $H$ be a simple graph. Then we have

$$
\operatorname{inj}(G, H)=\sum_{T \in \mathcal{G}(\leq k)}\left((-1)^{|V(G)|+|V(T)|} \sum_{P \in \mathcal{P}(G, T)}\|P\|\right) \operatorname{hom}(T, H) .
$$

To see this, let us consider the partially ordered set $\mathcal{P}(G)$ with respect to refinement. For $P, P^{\prime} \in \mathcal{P}(G)$ with $P^{\prime} \leq P$ (i.e. $P^{\prime}$ refines $P$ ), let $p_{1}, \ldots, p_{r}$ be a list of the $P$-classes and let $a_{i}$ be the number of $P^{\prime}$-classes contained in $p_{i}$ $(1 \leq i \leq r)$. Let $s=\sum_{i=1}^{r} a_{i}$ be the number of classes in $P^{\prime}$. Then the Mobius function is

$$
\mu\left(P^{\prime}, P\right)=(-1)^{r+s} \prod_{i=1}^{r}\left(a_{i}-1\right) !
$$

(see e.g. [10]). In particular, for the discrete partition $P_{0}$ we get

$$
\mu\left(P_{0}, P\right)=(-1)^{|V(G)|+|V(G / P)|}\|P\| .
$$

On the other hand, we have

$$
\operatorname{hom}\left(G / P^{\prime}, H\right)=\sum_{P^{\prime} \leq P \in \mathcal{P}(G)} \operatorname{inj}(G / P, H) .
$$

Now the Mobius inversion formula yields

$$
\operatorname{inj}(G, H)=\sum_{P \in \mathcal{P}(G)}(-1)^{|V(G)|+|V(G / P)|}\|P\| \operatorname{hom}(G / P, H)
$$

which, when collecting terms by $T=G / P \in \mathcal{G}(\leq k)$ gives the formula in Claim 2 .

The lemma follows from substituting the formula in Claim 2 into the formula in Claim 1 and collecting terms.

Now we show that the base parameters of a disconnected graph can be expressed as a convolution of the base parameters of its connected components, normalized by a constant computed from the multiplicities:

Lemma 3.2 Let $T$ be the disjoint union of the connected graphs $T_{1}, T_{2}, \ldots, T_{l}$. Let $S=\left\{j \mid \nexists i<j: T_{i} \cong T_{j}\right\}$ contain the indices of nonisomorphic $T_{j}$ 's and $m_{j}=\left|\left\{i \mid T_{i} \cong T_{j}\right\}\right|$ denote the multiplicity of $T_{j}$. Define $\sigma=\prod_{j \in S} m_{j} !$. Then for all $k>0$ we have

$$
c_{k}(T)=\frac{1}{\sigma} \sum_{\substack{\left(x_{1}, \ldots, x_{l}\right) \\ x_{1}+\cdots+x_{l}=k}} \prod_{j=1}^{l} c_{x_{j}}\left(T_{j}\right) .
$$


Proof. Recall that

$$
c_{k}(T)=\sum_{G \in \mathcal{G}(k)} \frac{(-1)^{|E(G)|+|V(G)|+|V(T)|+k}}{|\operatorname{Aut}(G)|} \sum_{P \in \mathcal{P}(G, T)}\|P\| .
$$

For a fixed $G$ and $P$, the connected components of $G / P$ can be identified with $T_{1}, T_{2}, \ldots, T_{l}$ in $\sigma$ possible ways. Each of these matchings gives a subdivision $G=G_{1} \cup G_{2} \cup \ldots \cup G_{l}$ by applying the inverse image of the quotient map to the $T_{i}$ 's. The restrictions $P_{i}=\left.P\right|_{G_{i}}$ of the partition $P$ satisfy $P_{i} \in \mathcal{P}\left(G_{i}, T_{i}\right)$ and

$$
\prod_{j=1}^{l}\left\|P_{j}\right\|=\|P\| \text {. }
$$

Therefore

$$
c_{k}(T)=\sum_{G \in \mathcal{G}(k)} \frac{(-1)^{|E(G)|+|V(G)|+|V(T)|+k}}{|\operatorname{Aut}(G)|} \sum_{G=G_{1} \cup \ldots \cup G_{l}} \sum_{\substack{P_{j} \in \mathcal{P}\left(G_{j}, T_{j}\right) \\ 1 \leq j \leq l}} \frac{1}{\sigma} \prod_{j=1}^{l}\left\|P_{j}\right\| .
$$

If we already know the isomorphism classes of $G_{1}, G_{2}, \ldots, G_{l}$, there are still

$$
\frac{|\operatorname{Aut}(G)|}{\prod_{j=1}^{l}\left|\operatorname{Aut}\left(G_{j}\right)\right|}
$$

possibilities to arrange them as a subdivision of $G$. It follows that $c_{k}(T)$ equals

$$
\sum_{\substack{\left(x_{1}, \ldots, x_{l}\right) \\ x_{1}+\ldots+x_{l}=k}} \sum_{\substack{G_{j} \in \mathcal{G}\left(x_{j}\right) \\ 1 \leq j \leq l}} \frac{|\operatorname{Aut}(G)|}{l} \cdot \frac{(-1)^{|E(G)|+|V(G)|+|V(T)|+k}}{|\operatorname{Aut}(G)|} \sum_{\substack{P_{j} \in \mathcal{P}\left(G_{j}, T_{j}\right) \\ 1 \leq j \leq l}} \frac{1}{\sigma} \prod_{j=1}^{l}\left\|P_{j}\right\| .
$$

By using

$$
|E(G)|+|V(G)|+|V(T)|+k=\sum_{j=1}^{l}\left(\left|E\left(G_{j}\right)\right|+\left|V\left(G_{j}\right)\right|+\left|V\left(T_{j}\right)\right|+x_{j}\right)
$$

and rearranging we get

$$
\begin{gathered}
c_{k}(T)=\frac{1}{\sigma} \sum_{\substack{\left(x_{1}, \ldots, x_{l}\right) \\
x_{1}+\ldots+x_{l}=k}} \prod_{j=1}^{l} \sum_{G_{j} \in \mathcal{G}\left(x_{j}\right)} \frac{(-1)^{\left|E\left(G_{j}\right)\right|+\left|V\left(G_{j}\right)\right|+\left|V\left(T_{j}\right)\right|+x_{j}}}{\left|\operatorname{Aut}\left(G_{j}\right)\right|} \sum_{P_{j} \in \mathcal{P}\left(G_{j}, T_{j}\right)}\left\|P_{j}\right\|= \\
=\frac{1}{\sigma} \sum_{\substack{\left(x_{1}, \ldots, x_{l}\right) \\
x_{1}+\ldots+x_{l}=k}} \prod_{j=1}^{l} c_{x_{j}}\left(T_{j}\right) .
\end{gathered}
$$

We can use the following variant of Lemma 3.2 when we would like to detach one connected component of $T$ at a time: 
Lemma 3.3 Let $T$ be the disjoint union of the connected graphs $T_{1}, T_{2}, \ldots, T_{l}$ where $l \geq 2$. Let $S=\left\{j \mid \nexists i<j: T_{i} \cong T_{j}\right\}$ contain the indices of nonisomorphic $T_{j}$ 's. Then we have

$$
k c_{k}(T)-\sum_{i=1}^{k-1} \sum_{j \in S} i c_{i}\left(T_{j}\right) c_{k-i}\left(T \backslash T_{j}\right)=0 .
$$

Proof. Let $m_{j}$ denote the multiplicity of $T_{j}$ and $\sigma=\prod_{j \in S} m_{j}$ ! as in Lemma 3.2 Since isomorphic $T_{j}$ 's have identical $c_{i}\left(T_{j}\right)$ and $c_{k-i}\left(T \backslash T_{j}\right)$, it follows that

$$
\sum_{j \in S} i c_{i}\left(T_{j}\right) c_{k-i}\left(T \backslash T_{j}\right)=\sum_{t=1}^{l} \frac{i}{m_{t}} c_{i}\left(T_{t}\right) c_{k-i}\left(T \backslash T_{t}\right) .
$$

By using Lemma 3.2 for $T$ and $\sigma$ and also for $T \backslash T_{t}$ and $\frac{\sigma}{m_{t}}$ we obtain:

$$
\begin{gathered}
k c_{k}(T)-\sum_{i=1}^{k-1} \sum_{j \in S} i c_{i}\left(T_{j}\right) c_{k-i}\left(T \backslash T_{j}\right)=k c_{k}(T)-\sum_{i=1}^{k-1} \sum_{t=1}^{l} \frac{i}{m_{t}} c_{i}\left(T_{t}\right) c_{k-i}\left(T \backslash T_{t}\right)= \\
=\frac{k}{\sigma} \sum_{\substack{\left(x_{1}, \ldots, x_{l}\right) \\
x_{1}+\ldots+x_{l}=k}} \prod_{j=1}^{l} c_{x_{j}}\left(T_{j}\right)-\sum_{i=1}^{k-1} \sum_{t=1}^{l} \frac{i}{m_{t}} \cdot \frac{m_{t}}{\sigma} \sum_{\substack{\left(x_{1}, \ldots, x_{l}\right) \\
x_{1}+\ldots+x_{l}=k \\
x_{t}=i}} \prod_{j=1}^{l} c_{x_{j}}\left(T_{j}\right)= \\
=\frac{1}{\sigma} \sum_{\substack{\left(x_{1}, \ldots, x_{l}\right) \\
x_{1}+\ldots+x_{l}=k}}\left(k-\sum_{t=1}^{l} x_{t}\right) \prod_{j=1}^{l} c_{x_{j}}\left(T_{j}\right)=0 .
\end{gathered}
$$

The last equation follows from $k-\sum_{t=1}^{l} x_{t}=0$.

Now we show that $p_{k}(H)$ can be expressed using the number of homomorphisms from connected graphs.

Theorem 3.4 Let $H$ be a simple graph on $n$ vertices and let $k>0$ be an integer. Then

$$
p_{k}(H)=\sum_{\substack{T \in \mathcal{G}(\leq k) \\ T \text { is connected }}}(-1)^{k-1} k c_{k}(T) \operatorname{hom}(T, H) .
$$

Proof. The Newton identites tell us that

$$
p_{k}(H)=(-1)^{k-1} k e_{k}(H)+\sum_{i=1}^{k-1}(-1)^{k-i-1} p_{i}(H) e_{k-i}(H)
$$

for all $k>0$. Using induction on $k$, we can assume that the result holds for all $j<k$. Lemma 3.1 gives us a formula for $e_{k}(H)$ in the parameters $c_{k}(T)$, 
namely we have

$$
e_{k}(H)=\sum_{T \in \mathcal{G}(\leq k)} c_{k}(T) \operatorname{hom}(T, H) .
$$

Using that hom is multiplicative (stated as Lemma 2.1) we get $p_{k}(H)$ as a fixed linear combination of the $\operatorname{hom}(T, H)$ 's. Let $q_{k}(T)$ denote the formal coefficient of hom $(T, H)$ in this sum. So, we have

$$
p_{k}(H)=\sum_{T \in \mathcal{G}(\leq k)} q_{k}(T) \operatorname{hom}(T, H) .
$$

This leads to the following equality for all $T$ :

$$
q_{k}(T)=(-1)^{k-1} k c_{k}(T)+\sum_{i=1}^{k-1}(-1)^{k-i-1} \sum_{\substack{U_{1} \in \mathcal{G}(\leq i) \\ U_{2} \in \mathcal{G}(\leq k-i) \\ U_{1} \cup U_{2}=T}} q_{i}\left(U_{1}\right) c_{k-i}\left(U_{2}\right)
$$

where $T$ is isomorphic to the disjoint union of $U_{1}$ and $U_{2}$.

Let $T \in \mathcal{G}(\leq k)$. We claim that

$$
q_{k}(T)=(-1)^{k-1} k c_{k}(T)
$$

if $T$ is connected and 0 otherwise. If $T$ is connected then it is impossible to choose $U_{1}$ and $U_{2}$ in the second sum above, so the claim holds. If $T$ is disconnected then as in Lemma 3.3 let $T$ be the disjoint union of the connected graphs $T_{1}, T_{2}, \ldots, T_{l}$ and let $S$ contain the indices of nonisomorphic $T_{j}$ 's. Using induction on $k$ we can assume that $q_{i}\left(U_{1}\right)=0$ unless $U_{1}$ is isomorphic to one of the $T_{j}$ 's. This gives

$$
q_{k}(T)=(-1)^{k-1} k c_{k}(T)+\sum_{i=1}^{k-1}(-1)^{k-i-1} \sum_{j \in S} q_{i}\left(T_{j}\right) c_{k-i}\left(T \backslash T_{j}\right) .
$$

We know from the induction hypothesis that $q_{i}\left(T_{j}\right)=(-1)^{i-1} i c_{i}\left(T_{j}\right)$ and therefore we get

$$
q_{k}(T)=(-1)^{k-1}\left(k c_{k}(T)-\sum_{i=1}^{k-1} \sum_{j \in S} i c_{i}\left(T_{j}\right) c_{k-i}\left(T \backslash T_{j}\right)\right)
$$

which is 0 according to Lemma 3.3 . 


\section{Convergence of chromatic measures}

In this section we prove Theorem 1.1. Theorem 1.2 and Proposition 1.3 For the convenience of the reader, we state the theorems again.

Theorem 1.1 Let $\left(G_{n}\right)$ be a Benjamini-Schramm convergent graph sequence of absolute degree bound $d$, and $\widetilde{D}$ an open neighborhood of the closed disc $\bar{D}$. Then for every holomorphic function $f: \widetilde{D} \rightarrow \mathbb{C}$, the sequence

$$
\int_{D} f(z) d \mu_{G_{n}}(z)
$$

converges.

Proof. We have

$$
\int_{D} z^{k} d \mu_{G}(z)=\frac{1}{|V(G)|} \sum_{i=1}^{|V(G)|} \lambda_{i}^{k}(G)=\frac{p_{k}(G)}{|V(G)|}
$$

for $k \geq 0$.

Since $f$ is holomorphic, it equals its Taylor series

$$
f(z)=\sum_{n=0}^{\infty} a_{n} z^{n}
$$

on an open neighborhood of $\bar{D}$. Let

$$
f_{k}(z)=\sum_{n=0}^{k} a_{n} z^{n}
$$

denote the partial sums. The $f_{k}$ 's converge uniformly on $D$, so we also know that

$$
F_{k}(G)=\int_{D} f_{k}(z) d \mu_{G}(z)=\sum_{n=0}^{k} a_{n} \int_{D} z^{n} d \mu_{G}(z)=\sum_{n=0}^{k} a_{n} \frac{p_{n}(G)}{|V(G)|}
$$

converges to

$$
F(G)=\int_{D} f(z) d \mu_{G}(z)
$$

uniformly on the set of graphs $G$ with $\mu_{G}$ supported on $D$. By Theorem 3.4 we have

$$
p_{n}(G)=\sum_{\substack{T \in \mathcal{G}(\leq n) \\ T \text { is connected }}}(-1)^{n-1} n c_{n}(T) \operatorname{hom}(T, G) .
$$


By rearranging, this gives

$$
F_{k}(G)=\sum_{T} b_{k, T} \frac{\operatorname{hom}(T, G)}{|V(G)|}
$$

where $T$ runs through connected graphs on at most $k+1$ vertices. Now let $G_{n}$ be a Benjamini-Schramm convergent sequence of graphs. By Lemma 2.2. the sequences

$$
\frac{\operatorname{hom}\left(T, G_{n}\right)}{\left|V\left(G_{n}\right)\right|}
$$

converge for every connected $T$. (Note that for non-connected $T$ this is in general false). This implies that $F_{k}\left(G_{n}\right)$ is convergent for every $k$. Since we already know that $F_{k}\left(G_{n}\right)$ uniformly converges to $F\left(G_{n}\right)$ for every $n$, we obtain that $F\left(G_{n}\right)$ is also convergent. It also follows that

$$
F\left(G_{n}, u\right)=\int_{D} f(z+u) d \mu_{G_{n}}(z)
$$

uniformly converges to a holomorphic function in a neighborhood of 0 .

We are ready to prove Theorem 1.2

Theorem 1.2 Let $\left(G_{n}\right)$ be a Benjamini-Schramm convergent graph sequence of absolute degree bound d with $\left|V\left(G_{n}\right)\right| \rightarrow \infty$. Then $\mathrm{t}_{G_{n}}(z)$ converges to a real analytic function on $\mathbb{C} \backslash \bar{D}$.

Proof. The principal branch of the complex logarithm function only takes values with an imaginary part in $(-\pi, \pi]$. Therefore $\varsigma_{G_{n}}(z)$ is always in the interval $\left(\frac{-\pi}{\left|V\left(G_{n}\right)\right|}, \frac{\pi}{\left|V\left(G_{n}\right)\right|}\right]$ and $\left|V\left(G_{n}\right)\right| \rightarrow \infty$ implies $\Im_{G_{n}}(z) \rightarrow 0$.

To prove convergence for the real part $\Re \mathrm{t}_{G_{n}}(z)$, consider a fixed $z_{0} \in \mathbb{C} \backslash \bar{D}$. Since the disc $B\left(z_{0}, C d\right)$ is bounded away from 0 , there exists a branch $\ln ^{*}$ of the complex logarithm function whose branch cut is a half-line emanating from 0 that is disjoint from the disc. It follows that $f(z)=\ln ^{*}\left(z_{0}-z\right)$ is holomorphic on an open neighborhood of $\bar{D}$. According to Theorem 1.1.

$$
\int_{D} \ln ^{*}\left(z_{0}-z\right) d \mu_{G_{n}}(z)
$$

converges uniformly in a neighborhood of $z_{0}$, which implies that

$$
\begin{aligned}
\Re \mathrm{t}_{G_{n}}\left(z_{0}\right)= & \frac{\Re \ln \operatorname{ch}_{G_{n}}\left(z_{0}\right)}{\left|V\left(G_{n}\right)\right|}=\frac{\sum_{\text {root }} \Re \ln \left(z_{0}-\lambda\right)}{\left|V\left(G_{n}\right)\right|}=\int_{D} \Re \ln \left(z_{0}-z\right) d \mu_{G_{n}}(z)= \\
& =\int_{D} \Re \ln ^{*}\left(z_{0}-z\right) d \mu_{G_{n}}(z)=\Re \int_{D} \ln ^{*}\left(z_{0}-z\right) d \mu_{G_{n}}(z)
\end{aligned}
$$


is locally uniformly convergent as a function of $z_{0}$. Since $\Re \ln \left(z_{0}-\lambda\right)$ is a harmonic function for all chromatic roots $\lambda$, so is $\Re \mathrm{t}_{G_{n}}\left(z_{0}\right)$, and the harmonicity of $\lim \mathrm{t}_{G_{n}}\left(z_{0}\right)=\lim \Re \mathrm{t}_{G_{n}}\left(z_{0}\right)$ follows from local uniform convergence. The observation that all harmonic functions are real analytic concludes the proof.

Now we prove Proposition 1.3 Note that already Salas and Sokal [1] showed that the pointwise limit of supports of $\mu_{T_{n}}$ is part of a particular algebraic curve. For convenience, we include some details on that, also adding a picture on the supporting set, but we do not introduce the transfer matrix method here. See [11] for a description of the transfer matrix method.

Proposition 1.3 The chromatic measures $\mu_{T_{n}}$ weakly converge.

Proof. We defined $T_{n}$ as the cartesian product of $C_{4}$ and $P_{n}$. By the transfer matrix method we obtain

$$
\operatorname{ch}_{T_{n}}(z)=v_{1} M^{n-1} \underline{\mathbf{1}}^{\top}
$$

with

$$
\begin{gathered}
v_{1}=\left(\begin{array}{ccc}
z^{4}-6 z^{3}+11 z^{2}-6 z & 2 z^{3}-6 z^{2}+4 z & z^{2}-z
\end{array}\right) \\
M=\left(\begin{array}{ccc}
z^{4}-10 z^{3}+41 z^{2}-84 z+73 & 2 z^{3}-14 z^{2}+38 z-40 & z^{2}-5 z+8 \\
z^{4}-10 z^{3}+40 z^{2}-77 z+60 & 2 z^{3}-13 z^{2}+32 z-29 & z^{2}-4 z+5 \\
z^{4}-10 z^{3}+39 z^{2}-70 z+48 & 2 z^{3}-12 z^{2}+26 z-20 & z^{2}-3 z+3
\end{array}\right) \\
\underline{\mathbf{1}}^{\top}=\left(\begin{array}{c}
1 \\
1 \\
1
\end{array}\right) .
\end{gathered}
$$

Using the eigenvectors of $M$ as our new basis we can diagonalize $M$ and rewrite the above expression as

$$
\operatorname{ch}_{T_{n}}(z)=u_{1} D^{n-1} u_{2}
$$

where

$$
\begin{gathered}
u_{1}=\left(\begin{array}{cc}
\frac{z^{7}-10 z^{6}+44 z^{5}-105 z^{4}+143 z^{3}-109 z^{2}+36 z+z^{3} r-2 z^{2} r+z r}{2 z^{3}-12 z^{2}+28 z-24} \\
\frac{z^{7}-10 z^{6}+44 z^{5}-10 z^{4}+143 z^{3}-109 z^{2}+36 z-z^{3} r+2 z^{2} r-z r}{2 z^{3}-12 z^{2}+28 z-24} \\
0
\end{array}\right)^{\top} \\
D=\left(\begin{array}{ccc}
\frac{z^{4}-8 z^{3}+29 z^{2}-55 z+46+r}{2} & 0 & 0 \\
0 & \frac{z^{4}-8 z^{3}+29 z^{2}-55 z+46-r}{2} & 0 \\
0 & 0 & 1
\end{array}\right) \\
u_{2}=\left(\begin{array}{c}
\frac{z^{4}-8 z^{3}+27 z^{2}-47 z+36+r}{2 r} \\
\frac{-z^{4}+8 z^{3}-27 z^{2}+47 z-36+r}{2 r} \\
0
\end{array}\right)
\end{gathered}
$$


and

$$
r=\sqrt{z^{8}-16 z^{7}+118 z^{6}-526 z^{5}+1569 z^{4}-3250 z^{3}+4617 z^{2}-4136 z+1776} .
$$

The matrix $D^{n-1}$ is straightforward to calculate, so we get the following closed formula for the chromatic polynomial:

$$
\operatorname{ch}_{T_{n}}(z)=a_{1} \lambda_{1}^{n-1}+a_{2} \lambda_{2}^{n-1}
$$

where

$a_{i}=\frac{z(z-1)\left(z^{4}-8 z^{3}+27 z^{2}-47 z+36+r_{i}\right)\left(z^{5}-9 z^{4}+35 z^{3}-70 z^{2}+73 z-36+z r_{i}-r_{i}\right)}{4 r_{i}(z-2)\left(z^{2}-4 z+6\right)}$

and

$$
\lambda_{i}=\frac{z^{4}-8 z^{3}+29 z^{2}-55 z+46+r_{i}}{2}
$$

with $r_{1,2}= \pm r$.

We are interested in the complex roots of this expression if $n$ is very large. We don't need to specify them exactly, but we'll prove a necessary condition. If the eigenvalues $\lambda_{i}$ differ in their absolute value for some $z$, there will be an arbitrarily large multiplicative gap between $a_{1} \lambda_{1}^{n-1}$ and $a_{2} \lambda_{2}^{n-1}$ for any values of $a_{i}$ unless both $a_{1} \lambda_{1}=0$ and $a_{2} \lambda_{2}=0$ holds.

It follows that all roots must have $\left|\lambda_{1}\right|=\left|\lambda_{2}\right|$ with the possible exception of a finite set consisting of the roots and singularities of $a_{1}, a_{2}, \lambda_{1}$ and $\lambda_{2}$, or equivalently, the roots of

$$
\begin{gathered}
z(z-1)(z-2)(2 z-5)\left(z^{2}-3 z+1\right)\left(z^{2}-4 z+6\right) . \\
\cdot\left(z^{6}-12 z^{5}+61 z^{4}-169 z^{3}+269 z^{2}-231 z+85\right) . \\
\cdot\left(z^{8}-16 z^{7}+118 z^{6}-526 z^{5}+1569 z^{4}-3250 z^{3}+4617 z^{2}-4136 z+1776\right) .
\end{gathered}
$$

Let's ignore this set $\mathcal{S}$ of special roots for now and concentrate on the general case of $\left|\lambda_{1}\right|=\left|\lambda_{2}\right|$ :

$$
\begin{gathered}
\lambda_{1} \overline{\lambda_{1}}=\lambda_{2} \overline{\lambda_{2}} \\
\frac{z^{4}-8 z^{3}+29 z^{2}-55 z+46+r}{2} \cdot \frac{\bar{z}^{4}-8 \bar{z}^{3}+29 \bar{z}^{2}-55 \bar{z}+46+\bar{r}}{2}= \\
=\frac{z^{4}-8 z^{3}+29 z^{2}-55 z+46-r}{2} \cdot \frac{\bar{z}^{4}-8 \bar{z}^{3}+29 \bar{z}^{2}-55 \bar{z}+46-\bar{r}}{2} \\
\left(z^{4}-8 z^{3}+29 z^{2}-55 z+46\right) \bar{r}+\left(\bar{z}^{4}-8 \bar{z}^{3}+29 \bar{z}^{2}-55 \bar{z}+46\right) r=0
\end{gathered}
$$

Our last expression means that $\left(z^{4}-8 z^{3}+29 z^{2}-55 z+46\right) \bar{r}$ is purely imaginary, which is equivalent to its square being a nonpositive real. When calculated, this gives a degree 14 algebraic curve clipped by a degree 16 algebraic curve, shown as the set $\mathcal{C}$ on the Figure 2 .

It follows that the curve $\mathcal{C}$ is compact, has an empty interior and its complement is connected. Hence the same holds for $\mathcal{C}^{\prime}=\mathcal{C} \cup \mathcal{S}$. 


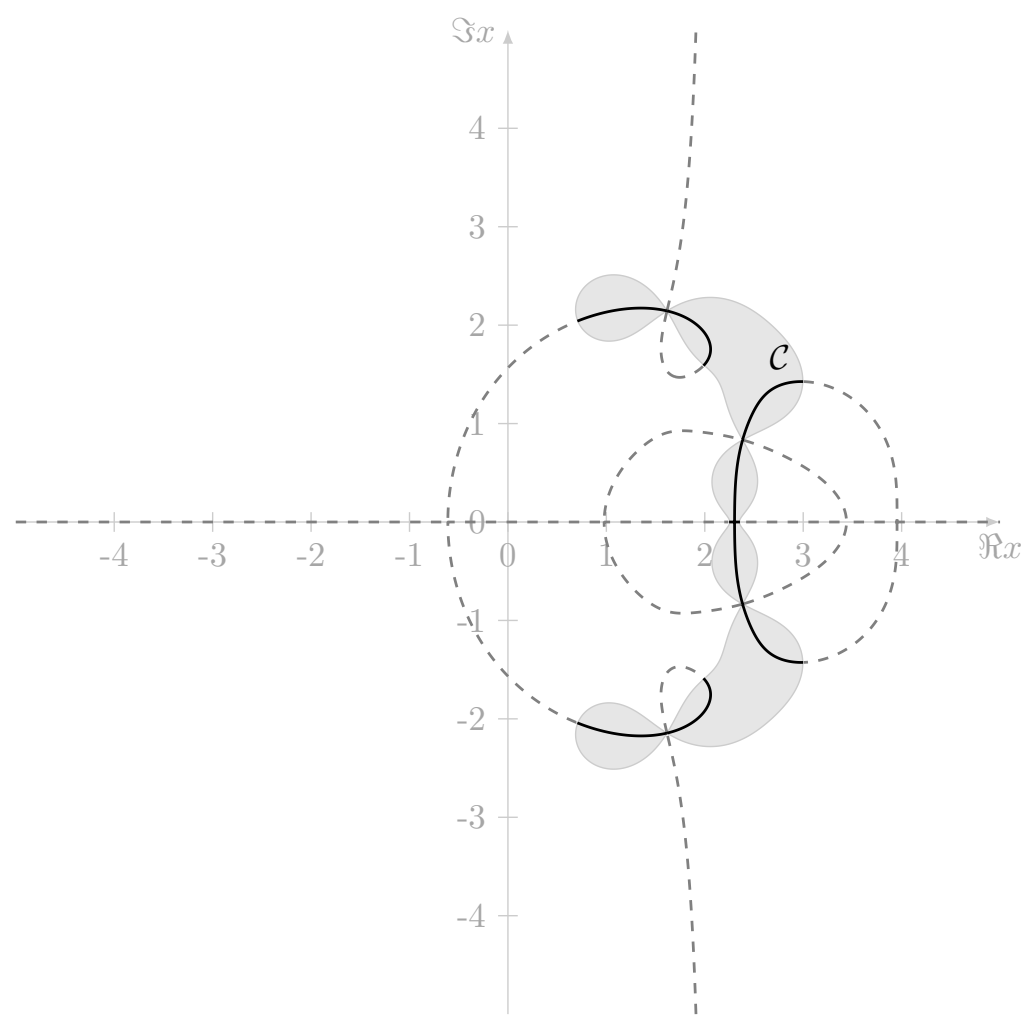

Figure 2: Possible limit points of chromatic roots of $T_{n}=C_{4} \times P_{n}$ as $n \rightarrow \infty$

Now Mergelyan's theorem 8 says that every continuous function on $\mathcal{C}^{\prime}$ can be uniformly approximated by polynomials. This implies that if two probability measures $\mu_{1}$ and $\mu_{2}$ are supported on $\mathcal{C}^{\prime}$ and the holomorphic moments satisfy

$$
\int_{\mathcal{C}^{\prime}} z^{k} d \mu_{1}(z)=\int_{\mathcal{C}^{\prime}} z^{k} d \mu_{2}(z) \quad(k \geq 1)
$$

then

$$
\int_{\mathcal{C}^{\prime}} f(z) d \mu_{1}(z)=\int_{\mathcal{C}^{\prime}} f(z) d \mu_{2}(z)
$$

for all continuous functions $f: \mathcal{C}^{\prime} \rightarrow \mathbb{R}$. Hence, we have $\mu_{1}=\mu_{2}$. Since any subsequential weak limit of $\mu_{T_{n}}$ is supported on $\mathcal{C}^{\prime}$, we get that $\mu_{T_{n}}$ is weakly convergent.

Remark. As we saw in the introduction, weak convergence does not hold in general. The phenomenon where an associated measure blows up by a small 
change of the graph but keeps its holomorphic moments unchanged also occurs in the spectral theory of directed graphs. Namely, the weak limit of the eigenvalue distributions of the directed path of length $n$ is the Dirac measure at 0 , while for the directed $n$-cycle the limit is the uniform measure on the unit circle centered at 0 . In both the chromatic and the spectral case, the reason is that the change only affects the coefficients of small index in the corresponding polynomial, and the $k$-th moment only depends on the $k$ highest index coefficients. It would be interesting to study this blow-up phenomenon using just abstract polynomials.

\section{Graphs of large girth}

In this section we study graphs with large girth and prove Theorem 1.4

Lemma 5.1 Suppose that the finite graphs $G$ and $H$ both have girth at least $g$ and $|E(G)|=|E(H)|$. Then $e_{i}(G)=e_{i}(H)$ holds for $i=0,1, \ldots, g-2$.

Proof. We use induction on $|E(G)|=|E(H)|$. If the number of edges is zero, the claim is trivial, as is when $g \leq 3$. Otherwise pick $e \in E(G)$ and $f \in E(H)$ arbitrarily and use the deletion-contraction argument:

$$
\begin{gathered}
e_{i}(G)=e_{i}(G \backslash e)+e_{i-1}(G / e) \\
e_{i}(H)=e_{i}(H \backslash f)+e_{i-1}(H / f)
\end{gathered}
$$

The claim follows from the observation that $G \backslash e$ and $H \backslash f$ have girth $\geq g$ while $G / e$ and $H / f$ have girth $\geq g-1$.

Lemma 5.2 Let $G$ be a finite graph with girth $\geq g$. Then $p_{i}(G)=|E(G)|$ for $i=1,2, \ldots, g-2$.

Proof. Let $H$ be an arbitrary tree on $|E(G)|+1$ vertices and use the previous lemma. Since the chromatic polynomial of $H$ is $q(q-1)^{|E(G)|}$, we have $e_{i}(H)=$ $\left(\begin{array}{c}|E(G)| \\ i\end{array}\right)$ for $i \leq|E(G)|$, which translates into $e_{i}(G)=\left(\begin{array}{c}|E(G)| \\ i\end{array}\right)$ for $i \leq g-2$. Substituting the $e_{i}$ 's into Newton's identities completes the proof.

Theorem 1.4 Let $G$ be a finite graph of girth $g$ and maximal degree $d$. Then for all $q>C d$ we have

$$
\left|\frac{\ln \operatorname{ch}_{G}(q)}{|V(G)|}-\left(\ln q+\frac{|E(G)|}{|V(G)|} \ln \left(1-\frac{1}{q}\right)\right)\right| \leq 2 \frac{(C d / q)^{g-1}}{1-C d / q} .
$$

Proof. The normalized log of the chromatic polynomial can be expanded as

$$
\begin{gathered}
\frac{\ln \operatorname{ch}_{G}(q)}{|V(G)|}=\int_{D} \ln (q-z) d \mu_{G}(z)=\ln q+\int_{D} \ln \left(1-\frac{z}{q}\right) d \mu_{G}(z)= \\
=\ln q-\sum_{n=1}^{\infty} \frac{1}{n q^{n}} \int_{D} z^{n} d \mu_{G}(z)
\end{gathered}
$$


where the Sokal bound $|z| \leq C d$ gives the constraint

$$
\left|\int_{D} z^{n} d \mu_{G}(z)\right| \leq(C d)^{n}
$$

for the holomorphic moments, and our last lemma implies

$$
\int_{D} z^{n} d \mu_{G}(z)=\frac{p_{n}(G)}{|V(G)|}=\frac{|E(G)|}{|V(G)|}
$$

for $n \leq g-2$. We also know that any real number $x \in[0,1)$ satisfies

$$
\sum_{n=g-1}^{\infty} \frac{x^{n}}{n}=\int_{0}^{x} \sum_{n=g-2}^{\infty} t^{n} d t=\int_{0}^{x} \frac{t^{g-2}}{1-t} d t \leq x \cdot \frac{x^{g-2}}{1-x}=\frac{x^{g-1}}{1-x} .
$$

Now we have

$$
\begin{gathered}
\left|\frac{\ln \operatorname{ch}_{G}(q)}{|V(G)|}-\left(\ln q+\frac{|E(G)|}{|V(G)|} \ln \left(1-\frac{1}{q}\right)\right)\right|= \\
=\left|\left(\ln q-\sum_{n=1}^{\infty} \frac{1}{n q^{n}} \int_{D} z^{n} d \mu_{G}(Z)\right)-\left(\ln q-\sum_{n=1}^{\infty} \frac{1}{n q^{n}} \cdot \frac{|E(G)|}{|V(G)|}\right)\right| \leq \\
\leq\left|\sum_{n=g-1}^{\infty} \frac{1}{n q^{n}}\left(\int_{D} z^{n} d \mu_{G}(z)-\frac{|E(G)|}{|V(G)|}\right)\right| \leq \sum_{n=g-1}^{\infty} \frac{1}{n q^{n}}\left(\left|\int_{D} z^{n} d \mu_{G}(z)\right|+\frac{|E(G)|}{|V(G)|}\right) \leq \\
\leq \sum_{n=g-1}^{\infty} \frac{(C d)^{n}+|E(G)| /|V(G)|}{n q^{n}} \leq \sum_{n=g-1}^{\infty} \frac{2(C d)^{n}}{n q^{n}} \leq 2 \frac{(C d / q)^{g-1}}{1-C d / q} .
\end{gathered}
$$

The theorem holds. 


\section{Appendix}

In the appendix we publish some data that may be useful for further analysis.

For abbreviation, we use the following terminology:

$$
\operatorname{hom}\left(\sum_{i=1}^{n} \alpha_{i} G_{i}, H\right)=\sum_{i=1}^{n} \alpha_{i} \operatorname{hom}\left(G_{i}, H\right)
$$

where the $G_{i}$ and $H$ are finite graphs.

One can express the first 4 chromatic coefficients as a linear combination of homomorphisms as follows:

$$
\begin{aligned}
& e_{0}(G)=\operatorname{hom}(\cdot, G) \\
& e_{1}(G)=\operatorname{hom}\left(\frac{1}{2} \bullet \bullet, G\right)
\end{aligned}
$$

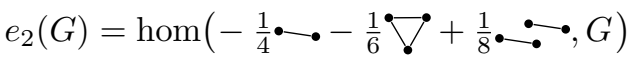

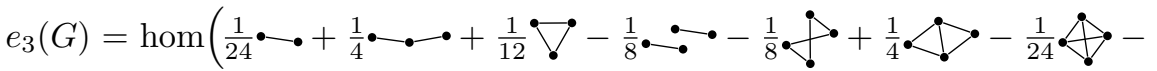

$$
\begin{aligned}
& \left.\frac{1}{12} \nabla \cdot \cdots+\frac{1}{48} \bullet \because, G\right)
\end{aligned}
$$

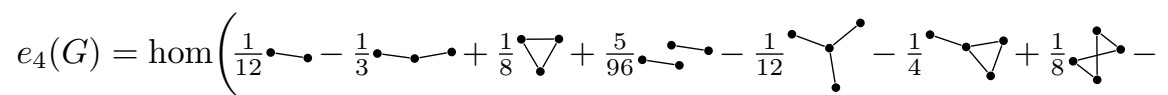

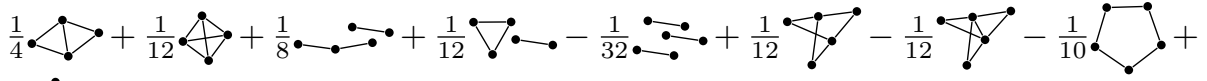

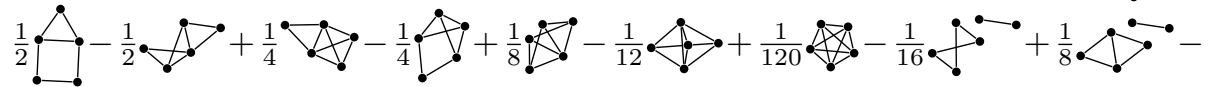

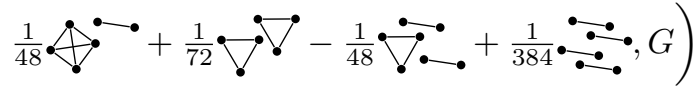

Also, one can express the first 5 chromatic moments as a linear combination of homomorphisms as follows.

$$
\begin{aligned}
& p_{0}(G)=\operatorname{hom}(\bullet, G) \\
& p_{1}(G)=\operatorname{hom}\left(\frac{1}{2} \bullet \bullet, G\right) \\
& p_{2}(G)=\operatorname{hom}\left(\frac{1}{2} \bullet \bullet+\frac{1}{3} \bigvee, G\right)
\end{aligned}
$$

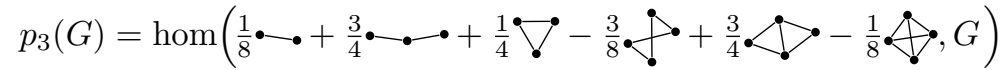

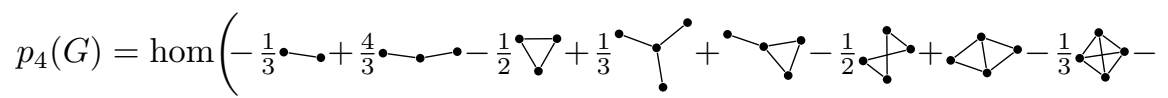

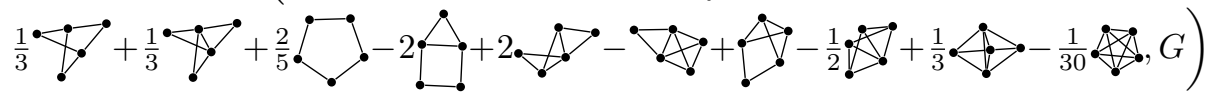




$$
\frac{25}{48}+\frac{35}{24}
$$




\section{References}

[1] A. Bandyopadhyay and D. Gamarnik, Counting without sampling. Asymptotics of the logpartition function for certain statistical physics models, Random Structures \& Algorithms 33 (2008)

[2] C. Borgs, Absence of Zeros for the Chromatic Polynomial on Bounded Degree Graphs, Combinatorics, Probability and Computing 15 (2006), 6374 .

[3] I. Benjamini and O. Schramm, Recurrence of distributional limits of finite planar graphs, Electron. J. Probab. 6 (2001), no. 23, 13 pp.

[4] C. Borgs, J. Chayes, J. Kahn and L. Lovász, Left and right convergence of graphs with bounded degree, Random Structures \& Algorithms 42 (2013)

[5] P. Csikvári and P. E. Frenkel, Benjamini-Schramm continuity of root moments of graph polynomials, http://arxiv.org/abs/1204.0463

[6] L. Lovász, Large Networks and Graph Limits. Colloquium Publications, vol. 60. American Mathematical Society (2012)

[7] R. Lyons, Asymptotic enumeration of spanning trees, Combinatorics, Probability and Computing 14 (2005), 491-522.

[8] S. N. Mergelyan, Uniform approximations to functions of a complex variable, Uspehi Mat. Nauk (N.S.) 7 (48) (1952), 31-122.

[9] A. Procacci, B. Scoppola and V. Gerasimov: Potts model on infinite graphs and the limit of chromatic polynomials. Commun. Math. Phys. 235 (2003), $215-231$.

[10] G. C. Rota, On the foundations of combinatorial theory I. Theory of Möbius functions, Probability theory and related fields 2 (4) (1964), 340-368.

[11] J. Salas and A.D. Sokal, Transfer Matrices and Partition-Function Zeros for Antiferromagnetic Potts Models V. Further Results for the Square-Lattice Chromatic Polynomial, J Stat Phys 135 (2009), 279-373.

[12] A. D. Sokal: Bounds on the complex zeros of (di)chromatic polynomials and Potts-model partition functions, Combinatorics, Probability and Computing 10 (2001), 41-77.

[13] A. D. Sokal, The multivariate Tutte polynomial (alias Potts model) for graphs and matroids, In: Webb, BS, (ed.) Surveys in Combinatorics, 2005, 173-226. Cambridge University Press 